Proceedings of ASME Turbo Expo 2019

Turbomachinery Technical Conference and Exposition

GT2019

June 17-21, 2019, Phoenix, Arizona, USA

GTP-19-1317

\title{
CONDITION MONITORING OF WIND TURBINE PLANETARY GEARBOXES UNDER DIFFERENT OPERATING CONDITIONS
}

\author{
Alexandre Mauricio \\ Shuangwen Sheng \\ Konstantinos Gryllias * \\ Depart. of Mechanical Engineering National Renewable Energy Laboratory \\ Depart. of Mechanical Engineering \\ KU Leuven \\ 15013 Denver West Pkwy \\ KU Leuven \\ Dynamics of Mechanical \\ Golden, Colorado, 80401, USA \\ Dynamics of Mechanical \\ and Mechatronic Systems, \\ Email: shuangwen.sheng@nrel.gov \\ and Mechatronics Systems, \\ Flanders Make \\ Flanders Make \\ Celestijnenlaan 300, BOX 2420, 3001, \\ Celestijnenlaan 300, BOX 2420, 3001, \\ Leuven, Belgium \\ Leuven, Belgium \\ Email: alex.ricardomauricio@kuleuven.be \\ Email: konstantinos.gryllias@kuleuven.be

\begin{abstract}
Digitally enhanced services for wind power could reduce Operations and Maintenance (O\&M) costs as well as the Levelised Cost Of Energy (LCOE). Therefore, there is a continuous need for advanced monitoring techniques which can exploit the opportunities of Internet of Things (IoT) and Big Data Analytics, revolutionizing the future of the energy sector. The heart of wind turbines is a rather complex epicyclic gearbox. Among others, extremely critical gearbox components which are often responsible for machinery stops are the rolling element bearings. The vibration signatures of bearings are rather weak compared to other components, such as gears, and as a result an extended number of signal processing techniques and tools have been
\end{abstract} \\ *Address all correspondence to this author.
}


proposed during the last decades, focusing towards accurate, early, and on time bearing fault detection with limited false alarms and missed detections. Envelope Analysis is one of the most important methodologies, where an envelope of the vibration signal is estimated usually after filtering around a frequency band excited by impacts due to the bearing faults. Different tools, such as Kurtogram, have been proposed in order to accurately select the optimum filter parameters (center frequency and bandwidth). Cyclic Spectral Correlation and Cyclic Spectral Coherence, based on Cyclostationary Analysis, have been proved as very powerful tools for condition monitoring. The monitoring techniques seem to have reached a mature level in case a machinery operates under steady speed and load. On the other hand, in case the operating conditions change, it is still unclear whether the change of the monitoring indicators is due to the change of the health of the machinery or due to the change of the operating parameters. Recently, the authors have proposed a new tool called IESFOgram, which is based on Cyclic Spectral Coherence and can automatically select the filtering band. Furthermore, the Cyclic Spectral Coherence is integrated along the selected frequency band leading to an Improved Envelope Spectrum. In this paper, the performance of the tool is evaluated and further extended on cases operating under different speeds and different loads. The effectiveness of the methodology is tested and validated on the National Renewable Energy Laboratory (NREL) wind turbine gearbox vibration condition monitoring benchmarking data set which includes various faults with different levels of diagnostic complexity as well as various speed and load operating conditions.

\section{NOMENCLATURE}

$\alpha$

$\tau$

$b w$

$c f$

$f$

$R_{x}$

$t$

$\mathbb{E}$

BPFI

BPFO

CSC

CSCor

EES

FTF
Cyclic frequency

Time lag

Bandwidth

Center frequency

Spectral frequency

Autocorrelation function

Time

Ensemble expected value

Ball Pass Frequency Inner Race

Ball Pass Frequency Outer Race

$\gamma_{x}(\alpha, f)$ Cyclic Spectral Coherence

$S_{x}(\alpha, f)$ Cyclic Spectral Correlation

Enhanced Envelope Spectrum

Fundamental Train Frequency 


$\begin{array}{ll}\text { GMF } & \text { Gear Mesh Frequency } \\ \text { HSS } & \text { High Speed Shaft } \\ \text { IES } & \text { Improved Envelope Spectrum } \\ \text { IESFOgram } & \text { Improved Envelope Spectrum via Feature Optimization-gram } \\ \text { IMS } & \text { Intermediate Speed Shaft } \\ \text { NREL } & \text { National Renewable Energy Laboratory } \\ \text { SES } & \text { Squared Envelope Spectrum } \\ \text { PLC } & \text { Planet Carrier }\end{array}$

\section{INTRODUCTION}

Wind power is one of the fastest-growing renewable energy technologies and its usage is rising worldwide, partially due to the falling of cost. The global installed wind-generation capacity onshore and offshore has increased by almost a factor of 50 during the last two decades, climbing from $7.5 \mathrm{GW}$ in 1997 to $487 \mathrm{GW}$ by 2016 [1]. The production of wind electricity has been doubled between 2009 and 2013 while the offshore wind power offers a tremendous potential. On the other hand, there is an emerging need for innovative solutions and technologies to increase the efficiency and optimization of renewable energy portfolios based on an advanced asset management. Therefore, wind energy is an emerging market for power generation, becoming more cost effective in the last years. Moreover, there is still a need for the further reduction of the cost of the wind turbines. One of the strategies lies in extending wind turbine lifetime use and in reducing the maintenance cost by monitoring the condition of the wind turbines $[2,3]$. The analysis of temperature, vibration, and acoustic signals by using advanced signal processing tools provides insight into the health condition of rotating machinery and early detection of damage, thereby avoiding unexpected breakdown of the components and increasing the generated power-to-cost ratio.

Along with the reduction of cost for sensors, the data acquisition systems and the increased computing power achieved in the last decades, several advanced signal processing tools have been proposed towards condition monitoring of rotating machinery with specific focus on gears, bearings, shafts, and other rotating machinery components which are key components of complex machinery, such as wind turbines [4]. One of the most widely used approaches for bearing diagnostics is the Squared Envelope Spectrum (SES) [5]. The method is based on the demodulation of the raw vibration signal in order to reveal the harmonics of the characteristic bearing frequencies that may indicate damage (i.e., BPFO, BPFI, etc.). Usually, the bearing signature is weaker than its deterministic counterparts (i.e., gears, shafts, etc.), and thus the bearing modulations are hidden in the spectrum. In this case, it is common to apply a band-pass filter to the signal around the resonant frequencies of the structure. Theory dictates that the resonant frequencies are usually excited by the impulses due to the bearing fault, and filtering around this band enhances the Signal-to-Noise Ratio (SNR) and provides a clearer SES [6], as demodulation methods, such as the Hilbert Transform, are sensitive in noise. The selection of the filter parameters can be made based on engineering experience or by using automated selection tools, such as the Kurtogram [7], the ICSgram [8] and the Peak Energy 
Criterion [9], to name a few. These tools are based on the optimization of a defined feature.

Apart from the SES-based methods, cyclostationary tools for condition monitoring, namely the Cyclic Spectral Correlation (CSC) and Coherence (CSCoh), have gained increasing interest. These tools show a good performance in describing first-order and secondorder cyclostationary signals. In other words, CSCoh and CSC are shown to be robust in the extraction and description of hidden periodicities, such as bearing signals [10,11]. Their application leads to a bivariable map in the frequency-frequency domain, where one axis describes the carrier signal content and the other axis describes the cyclic modulation signal. The integration of these bivariable maps shows good capabilities in generating enhanced demodulated spectra that exhibit the hidden characteristic frequencies. Their integration can be blind or selective, demonstrating that there is a good potential on the methods for bearing diagnostics, even though little research has been performed when compared to the SES.

The National Renewable Energy Laboratory (NREL) performed a round robin condition monitoring experiment on wind turbine gearboxes in order to test the capabilities of vibration analysis [12]. Various industrial and academic entities participated in the blind analysis to contribute to the condition monitoring field, and to further develop and validate methodologies that would increase the detection rate of damaged components on a gearbox of a wind turbine [4]. One of the existing faults, located at the low speed shaft could not be detected [13] until last year, when a new signal processing method based on cyclostationarity was proposed [14]. However, the research on this paper was focused on one data set measured at one speed and load condition and does not take into account measurements under different speed and load operating conditions.

The aim of this paper is to further extend the research on cyclostationary-based tools for bearing diagnostics on wind turbine gearboxes operating under different cases of load and speed conditions. The methodology bases itself on the IESFOgram, which has been proposed by the authors as a band selection tool for the integration on the CSCoh bivariable map in order to detect the diagnostic information. The methodology is applied and validated on the NREL wind turbine gearbox data sets, focusing on bearing diagnostics. The main novelty of the paper lies on the application, the validation and the analysis of the detection performance of the IESFOgram on signals, captured on wind turbines, operating under different speed and load conditions. Furthermore, the band selection criterion of IESFOgram has been improved, taking into account a normalization of the feature by the harmonics noise background level, as will be described later.

The rest of the paper is organised as follows. The theory of cyclostationary tools and the IESFOgram are first briefly presented. Then the NREL wind turbine gearbox setup is presented and the three measured cases under different operating conditions are detailed. Moreover, the method is applied to the data sets with the objective of validation and testing and its results and performance are discussed. Finally, some conclusions are drawn at the end of the paper. 


\section{INTEGRATION OF THE CYCLIC SPECTRAL COHERENCE}

Bearing diagnostics is a field of intensive research, due to the fact that bearings are critical machine element components of rotating machinery. In a complex system, the vibration signatures of the bearings are usually weak and masked by other components, such as gears. Therefore fault detection and diagnosis is often a difficult task.

A classical signal processing method for bearing fault detection and diagnosis is the "High frequency resonance technique", which was called later "Envelope Analysis". The generation of localised faults on the inner or the outer race of a bearing leads to the generation of impulses, each time the rolling element passes over the defect. The series of impulses excite the structural resonance frequencies, which are finally modulated by the characteristic bearing fault frequencies. Hilbert Transform is often used for the demodulation of the modulated signals but its performance depends on the SNR as it is very sensitive to noise. Therefore, usually as a pre-step a band pass filter is used, filtering the signal around an excited frequency band where the SNR is high. Afterwards, the filter signal is demodulated and the SES is extracted. The SES is further analysed and the defect is recognised by the presence of clear peaks at the harmonics of the characteristic bearing fault frequency. The selection of the characteristics of the filter needs expertise or the application of a dedicated tool, such as Kurtogram. Kurtogram has been proposed as a powerful tool for the selection of the optimum frequency band based on the maximisation of the Spectral Kurtosis but unfortunately it is sensitive to outliers. Localised bearing faults give rise to signals that can be processed as cyclostationary. A signal is characterised as cyclostationary of order $\mathrm{n}$ if its nth order statistics are periodic. Therefore, a first-order cyclostationary signal (CS1) has a periodic mean value (e.g., a periodic signal plus noise), whereas a second-order cyclostationary signal (CS2) has a periodic variance (e.g., an amplitude modulated white noise). The autocorrelation function $R_{2 x}(\tau, t)$ of CS2 signals is a periodic function with a period $T$ as described by Eq. 1:

$$
R_{2 x}(\tau, t)=R_{2 x}(\tau, t+T)=\mathbb{E}\left\{x(t) x(t-\tau)^{*}\right\}
$$

where $x(t)$ is a signal sampled at constant time intervals, $\mathbb{E}$ is the ensemble average, and $\tau$ is the time lag [6]. The statistics are calculated by ensemble averaging over an ensemble of realisations.

\section{Cyclic Spectral Coherence}

The CSCoh is a recently proposed tool that is based on the CSC, where the signal modulations and the carriers are correlated according to the definition of second-order of cyclostationarity. The CSC can be described by Eq. 2: 


$$
\operatorname{CSC}_{x}(\alpha, f)=\lim _{T \rightarrow \infty} T^{-1} \mathbb{E}\left[\mathscr{F}^{T}\{x(t)\}^{*} \mathscr{F}^{T}\{x(t-\tau)\}\right]
$$

where $\mathscr{F}^{T}\{x(t)\}$ is the Fourier Transform of the signal $x(t)$ and $*$ stands for the conjugate transpose. The processing of the CSC reveals the hidden modulations as high correlation values at which the carrier frequency, $f$, is modulated by the cyclic frequency, $\alpha$. The expression measures the correlation level of the signal between two frequency components of the signal at $f$ and $f+\alpha_{t}[15]$, by obtaining the ensemble expected value, $\mathbb{E}$, of the Fourier Transform at different time lags.

In order to minimize uneven distributions, a whitening operation can be applied to the CSC. This extended tool, named the CSCoh, describes the spectral correlations in normalized values between 0 and 1 and is defined by Eq. 3:

$$
\operatorname{CSCoh}_{x}(\alpha, f)=\frac{\operatorname{CSC}_{x}(\alpha, f)}{\sqrt{\operatorname{CSC}_{x}(0, f) C S C_{x}(0, f-\alpha)}}
$$

Both the CSC and the CSCoh result in bivariable maps which highlight the modulation frequencies in the $\alpha$ axis and their frequency content, $f$. CSCoh is used in the frames of this paper as it performs better than the CSC for fault detection [11].

\section{Improved Envelope Spectrum via Feature Optimization -gram}

As mentioned before, the SES is often estimated after band-pass filtering of the signal, resulting in enhanced demodulated frequencies plotted on the spectrum for analysis, assuming that a correct band for filtering has been selected. The Improved Envelope Spectrum (IES) is equivalent to the SES in this aspect, where a band of carriers is integrated and the corresponding demodulated frequencies are present in the final spectrum. It can be defined as in Eq. 4:

$$
\operatorname{IES}(\alpha)=\frac{1}{F_{2}-F_{1}} \int_{F_{1}}^{F_{2}}\left|\operatorname{CSCoh}_{x}(\alpha, f)\right| d f
$$

where $F_{1}$ is the low limit and $F_{2}$ is the high limit of the band, respectively. As can be implied from the equations, the IES (IES( $\left.\alpha\right)$ ) represents the modulation frequencies (or cyclic frequencies) present in the processed signals. The IES is expected to provide a better 
enhancement of the cyclic components, when compared to the classical SES, due to the ability of the CSCoh in extracting second-order cyclostationarity information.

For all the possible combinations of the characteristic frequencies present in the signal, there is one optimal band of frequencies between 0 and the Nyquist frequency, which optimizes the specific characteristic frequency of interest.

The main focus of the proposed method is to propose a tool for the correct selection of the optimal band of frequencies that maximises the amplitude of the fault frequencies and therefore results in a spectrum that is easy to be analysed and to lead to a clear fault diagnosis. This is done through the optimization of a feature based on the cyclic frequency domain. The feature in this paper is defined as the sum of 3 harmonics of the BPFO or BPFI, normalized by the background noise around each of the three harmonics. The feature feat $(c f, b w)$ is described in Eq. 5:

$$
f e a t(c f, b w)=\sum_{k=1}^{N} \frac{I E S\left(k f_{\text {fault }}\right)}{\frac{1}{2 f_{b}}\left[\int_{k f_{\text {fault }}-f_{b}}^{k f_{\text {faul }}+f_{b}} \operatorname{IES}(\alpha) d \alpha-I E S\left(k f_{\text {fault }}\right)\right]}
$$

where $f_{\text {fault }}$ is the fault frequency of interest corresponding to the BPFO or the BPFI in this paper.

The method begins by obtaining the CSCoh and performing a series of filters with a set combination of bandwidth, $b w$, and center frequency, $c f$. Each filter corresponds to a single $\operatorname{IES}(\alpha)$ and feature value $f e a t(c f, b w)$. After calculating the full series of IES and finishing the iteration, all the feature values are displayed in a 1/3 binary tree similar to the Fast Kurtogram. The band that maximizes the feature value is the one that is selected for the integration of the CSCoh, therefore obtaining the Improved Envelope Spectrum with the optimal feature. The methodology is clearly presented in Fig. GTP-19-1317-Fig01.

\section{OVERVIEW OF THE WIND TURBINE GEARBOX}

In order to test and validate its performance, the proposed method is applied on an experimental data set provided by NREL. The setup is a three-bladed wind-turbine with a rated power of $750 \mathrm{~kW}$, as is presented in Fig. GTP-19-1317-Fig02. The wind turbine blades rotate at low speeds and the speed is increased by the use of a gearbox in order to optimize the power output of the generator. The wind turbine planetary gearbox contains a planetary stage and two conventional ones, including four shafts with increasing speed ratio. The slowest shaft is the Planetary Carrier (PLC), which is the input to the planetary gearbox connected to the Low Speed Intermediate Shaft (LSS), which is the output of the planetary stage. This, in turn, is connected to the Intermediate Speed Stage by the first conventional stage, increasing the speed of the Intermediate Speed Shaft (IMS). The last conventional high speed stage further increases the speed of the High Speed Shaft (HSS), that is connected to the generator, which is also the gearbox final output. The diagram of the gearbox is shown in Fig. GTP-19-1317-Fig03 which also depicts the nomenclature used in this paper. 
Two gearboxes of the same design, one in healthy state and one in damaged state, were used to collect four data sets. The damaged gearbox contains various gear and bearing faults in different stages. The measurements were obtained under four conditions, which correspond to one healthy case and to three (3) fault cases under different speed and load conditions. The speed and power (percentage of rated power) specifications for each case are described in Table 1.

The frequencies of interest are analytically described in the round robin [4] as orders function of HSS and based on them the various gear related frequencies were calculated and are presented in orders in Table 2. The sensors (accelerometers) were placed on various locations on the outside of the gearbox and the signals were sampled at $40 \mathrm{kHz}$ per channel. There are eight sensors in total, and the labelling is indexed from sensor AN3 to sensor AN10, where the AN3 and AN4 are mounted onto the ring gear, closer to the gearbox components with the lower speed. The sensor AN5 is near to the LSS, the sensor AN6 is near to the IMS, and the sensors AN7 to AN9 are close to the HSS. Therefore, the labels correspond to the component speeds and the lower indices correspond to lower rotating speeds. This labelling is followed in [4] and therefore it is assumed in this paper as well. The study of this paper focuses on bearing faults and therefore the gear faults have not been considered and analysed. The various detectable bearing faults and their characteristic frequencies are presented in Table 3 for the case of $1200 \mathrm{rpm}$ operating speed and in Table 4 for the cases of $1800 \mathrm{rpm}$ operating speed. The data sets were extensively analysed in a round robin by various partners [13] and the findings were published by NREL [4]. The wind turbine data sets, the kinematics, and the faults are also detailed by NREL in [12].

\section{RESULTS AND DISCUSSION}

The sensors are placed on different locations outside of the gearbox and the damage content of the bearing faults is better noticeable by the specific sensor which is closer to the faulty bearing. As a consequence, some faults are detected more clearly in some sensors; therefore the correct sensor data must be selected beforehand. As an example, the sensor AN7 is located near the HSS downwind bearings, thus it captures better the damage on this bearing compared to the other sensors.

\section{CASE C}

The first step in the processing scheme is to perform the CSC and display the bivariable map. The most prominent of all the bearing fault frequencies is the HSS downwind bearing's BPFI based on the analysis of the signal of sensor AN7 for the damaged case. The various partners of the round robin study reached this consensus, declaring this fault signature to be easier detected compared to the others. The bearing fault characteristic frequency and its carrier frequency can be seen clearly in the damaged case, whereas it is not present, as normally expected, in the healthy case. The CSC map zoomed around the BPFI for the healthy case and the damage case under the same operating conditions (case C) are demonstrated in Fig. GTP-19-1317-Fig04.

The CSC map shows that the carrier frequencies modulated by the BPFI are present in the frequency band between $10 \mathrm{kHz}$ and 15 kHz. Performing the integration of the CSC over this band should provide a clear detection of the fault. Selecting the BPFI harmonics 
as the feature to be optimized, the IESFOgram should also result in a band between these frequencies. Indeed, as expected, the optimal band selected by the IESFOgram corresponds to the high frequency content of the spectrum, with a center frequency, equal to $c f=12.5$ kHz, as presented in Fig. GTP-19-1317-Fig05.

The same results are achieved for all five bearing fault types present in the gearbox. The CSCoh analysis highlights the presence of cyclic frequencies corresponding to the bearing characteristic frequencies when there is indeed a damage. In contrast, the fault frequencies are not detectable in the healthy case. In the healthy case where there is no damage in the bearing, the band selection tool does not optimize the feature but practically optimises the sum of the amplitudes of three random frequency components at the vicinity of the nominal characteristic bearing fault frequency. The procedure returns a different band of frequencies, usually in the low band of the frequency spectrum, where a multitude of deterministic frequencies related to the gears and shafts is always present and dominant in the absence of the fault. A more detailed analysis comparing the healthy case and the damaged case $\mathrm{C}$ has been presented in [14]. There, the band selection tool, which is the precursor of the IESFOgram, is shown to select the optimal integration band and all the bearing fault frequencies are correctly detected on the corresponding spectra estimated after the processing of the signals captured by the sensors at the positions AN5, AN6, and AN7. It should be noted that the methodology can detect successfully even the BPFO at the PLC, which seems to be the most complicated case for diagnosis from this data set. The IESFOgram has been applied on a signal captured by the AN5 sensor and the corresponding IES is presented at Fig. GTP-19-1317-Fig06, thereby focusing to the detection of the PLC fault. Analysing carefully the IES it can be concluded that the 1st, 2nd and 3rd harmonics of the BPFO are clearly identified. Moreover, it should be noted that the peak at the first harmonic of BPFO does not coincide with the harmonic of the sun shaft which is very close.

\section{CASE B}

The measurements in Case B have been captured operating at $1800 \mathrm{rpm}$ and at $50 \%$ rated power as presented in Table 1. For this case, most of the defects are detected using the IESFOgram. The IES of the HSS DW is presented in Fig. GTP-19-1317-Fig07. The 3 harmonics of the Fundamental Train Frequency (FTF) are clearly identified.

On the other hand, the detection of the PLC UW BPFO at this case where the load is lower seems to be uncertain, although it was clearly detected in case C. The analysis of the CSCoh does not return a clear peak at the BPFO cyclic frequency and the IESFOgram does not show a convergence to an optimal band, as seen in Fig. GTP-19-1317-Fig08.

This is a typical IESFOgram resulting from a healthy case. As the fault signature is not present in the CSCoh, the IESFOgram tries to optimize peaks at the background noise, resulting in random narrow bands with high feature value. The IES for the PLC UW BPFO is shown in Fig. GTP-19-1317-Fig09. Some peaks are present at the harmonics of the BPFO at the IES, but the presence/pollution of several peaks along the spectrum makes the diagnosis debatable. As such, the fault is considered not detected for this case, especially due to its non-existence on the CSCoh. It has been already demonstrated that this fault case is the most complicated case to detect in this data set but the low load operating conditions seem to turn their detection even more complicated. 
One explanation for this phenomenon is that the low load decreases the fault impulses. According to the theory [6], high loads applied on the structure increase the impulsive strength of the bearing faults; therefore, for low load cases the damaged bearing signature on the signal decreases lowering its detectability in real cases $[8,16]$.

\section{CASE A}

For the only case measured at the lower speed of $1200 \mathrm{rpm}$, the detection rate of the faults would be expected to decrease, due to its lower frequency and therefore lower number of fault impulses for the same sampling frequency. However, the method shows no problem on detecting all the fault frequencies at the spectra. As can be seen in Fig. GTP-19-1317-Fig10, the IESFOgram shows a good convergence of high feature values in the high frequency bands and the IES shows clearly 3 harmonics of the PLC UW BPFO. Even though the speed decreased, as the case corresponds to high load, the impulse strength of the faults is assumed to have remained high and thus the same diagnosis performance of case $\mathrm{C}$ is achieved on case A. All damages for the characteristic frequencies described in Table 2 are clearly detected.

\section{Performance analysis}

The measurement campaign contains 10 measured signals per sensor and per case. Analysing the spectra for each of the 3 cases and each of the 5 bearing damages, the results of the performance analysis described in Table 5 can be extracted.

From here, it can be seen that all damage cases are easily detected in 10 or 9 of the 10 available signals, resulting in a detection rate of $100 \%$ and $90 \%$, respectively, with the exception of the BPFO of the planet stage. The highest detection rate for this fault occurs at the operating conditions of case $\mathrm{C}$, which is to be expected as case $\mathrm{C}$ corresponds to the highest speed and load case. For the low load, no peaks at the BPFO were detected with confidence, while for the low speed the peaks are clear on 5 out of the 10 signals. Additionally it should be noted that the spectra presented in this paper show a dotted line which corresponds to a threshold equal to 6 times the moving absolute deviation above the moving median, and was used by the authors to determine that any peak below the threshold does not correspond to a valid one.

\section{CONCLUSIONS}

The performance of a cyclostationary-based approach has been evaluated for bearing diagnostics of a planetary gearbox under different operating speed and load conditions. The methodology is based on the Cyclic Spectral Coherence (CSCoh) and on the Improved Envelope Spectrum via Feature Optimisation-gram (IESFOgram), which has been recently proposed as an automated band selection tool for the optimal band of integration on the CSCoh bivariable map, leading to an Improved Envelope Spectrum. The method is tested and validated on a wind turbine data set provided by National Renewable Energy Laboratory (NREL) under 3 different combinations of speed and load operating conditions. The proposed methodology is successful in detecting almost all bearing defects generated on the 
wind turbine gearbox. As stated by the initial report of NREL, one of the defects had not been identified by the analysis done by a number of industrial partners during the benchmarking activity. On the other hand, IESFO without any additional preprocessing steps detect successfully the defect in two out of three cases. The third case corresponds to the gearbox operation under the lowest load condition. On the other hand, the damage is detected in the higher load cases, indicating that the torque plays an important role in the detectability of the defects. The research in this paper and the literature concludes that the higher load may increase the impulsiveness strength of hard to detect defects and that torque plays a role in the detectability of defects. As a final conclusion, the IESFOgram appears to be a strong tool for fault detection and diagnosis, and presents high performance and detectability. In this framework, the authors will continue working on the topic focusing toward the extension of the methodology in order to remove the influence of speed and load in all the cases.

\section{ACKNOWLEDGMENT}

K. Gryllias would like to gratefully acknowledge the Research Fund KU Leuven. The authors would like to thank the National Renewable Energy Laboratory for organizing the test on condition monitoring of the wind turbine gearbox and providing the resulting data sets. This work was authored [in part] by the National Renewable Energy Laboratory, operated by Alliance for Sustainable Energy, LLC, for the U.S. Department of Energy (DOE) under Contract No. DE-AC36-08GO28308. Funding provided by the U.S. Department of Energy Office of Energy Efficiency and Renewable Energy Wind Energy Technologies Office. The views expressed in the article do not necessarily represent the views of the DOE or the U.S. Government. The U.S. Government retains and the publisher, by accepting the article for publication, acknowledges that the U.S. Government retains a nonexclusive, paid-up, irrevocable, worldwide license to publish or reproduce the published form of this work, or allow others to do so, for U.S. Government purposes.

\section{REFERENCES}

[1] IRENA, 2019. International Renewable Energy Agency wind energy description, https://www.irena.org/wind.

[2] Scholbrock, A., Fleming, P., Wright, A., Wang, N., Schlipf, D., and Johnson, K., 2016. "Lidar-enhanced wind turbine control: Past, present, and future”. In Proceedings of the 2016 American Control Conference Boston, Massachusetts, Academic Press, pp. 1-8.

[3] Lebold, M., McClintic, K., Campbell, R., Byington, C., and Maynard, K., 2000. "Review of vibration analysis methods for gearbox diagnostics and prognostics". In Proceedings of the 54th Meeting of the Society for Machinery Failure Prevention Technology, Academic Press, pp. 623-634.

[4] Sheng, S., 2012. "Wind turbine gearbox condition monitoring round robin study - vibration analysis". NREL/TP-5000-54530.

[5] McFadden, P., and Smith, J., 1984. "Vibration monitoring of rolling element bearings by the high-frequency resonance technique — a review". Tribology International, 17(1), pp. 3 - 10. 
[6] Randall, R. B., and Antoni, J., 2011. "Rolling element bearing diagnostics — a tutorial". Mechanical Systems and Signal Processing, 25(2), pp. $485-520$.

[7] Randall, R. B., and Sawalhi, N., 2011. Use of the cepstrum to remove selected discrete frequency components from a time signal. Springer New York, New York, NY, pp. 451-461.

[8] Smith, W., Fang, Z., Peng, Z., and Randall, R., 2016. “Optimised spectral kurtosis for bearing diagnostics under electromagnetic interference". Mechanical Systems and Signal Processing, 75, pp. $371-394$.

[9] Gryllias, K., and Antoniadis, I., 2009. "A peak energy criterion for the selection of resonance bands in complex shifted morlet wavelet (csmw) based demodulation of defective rolling element bearings vibration response". International Journal of Wavelets, Multiresolution and Information Processing, 7(4), p. 387-410.

[10] Antoni, J., 2007. "Cyclic spectral analysis in practice”. Mechanical Systems and Signal Processing, 21(2), pp. 597 - 630.

[11] Antoni, J., Xin, G., and Hamzaoui, N., 2017. "Fast computation of the spectral correlation". Mechanical Systems and Signal Processing, 92, pp. $248-277$.

[12] Sheng, S., 2012. "Wind turbine gearbox vibration condition monitoring benchmarking datasets". NREL/TP-5000-54530.

[13] Sheldon, J., Mott, G., Lee, H., and Watson, M., 2014. “Robust wind turbine gearbox fault detection”. Wind Energy, 17(5), pp. 745-755.

[14] Mauricio, A., Qi, J., and Grylllias, K., 2018. "Vibration based condition monitoring of wind turbine gearboxes based on cyclostationary analysis". Journal of Engineering for Gas Turbines and Power, 141(3).

[15] Abboud, D., Baudin, S., Antoni, J., Rémond, D., Eltabach, M., and Sauvage, O., 2016. “The spectral analysis of cyclo-nonstationary signals". Mechanical Systems and Signal Processing, 75, pp. $280-300$.

[16] Mauricio, A., Qi, J., Gryllias, K., Sarrazin, M., Janssens, K., Smith, W., and Randall, R., 2018. "Cyclostationary based bearing diagnostics under electromagnetic interference". 25th International Congress on Sound and Vibration. 


\section{Tables:}

TABLE 1: Power and speed details of the 4 measurement cases.

\begin{tabular}{lcc}
\hline Case & Speed (rpm) & Rated Power $(\%)$ \\
\hline Healthy & 1800 & 100 \\
Case C & 1800 & 100 \\
Case B & 1800 & 50 \\
Case A & 1200 & 100 \\
\hline
\end{tabular}

TABLE 2: Shafts and gears orders.

\begin{tabular}{lc}
\hline Gears & Order (/HSS) \\
\hline Rotor & 0.012 \\
Planets & 0.043 \\
Sun Shaft & 0.070 \\
Intermediate Shaft & 0.250 \\
Generator Shaft (HSS) & 1.000 \\
(GMF) Sun $\rightarrow$ IMS & 5.750 \\
(GMF) IMS $\rightarrow$ HSS & 22.000 \\
\hline GMF, Gear Mesh Frequency; HSS, High \\
Speed Shaft; IMS, Intermediate Speed \\
Shaft.
\end{tabular}


TABLE 3: Damaged bearings and their corresponding frequencies for case A (1200 rpm).

\begin{tabular}{lccr}
\hline Bearing label & Fault type & Frequency $(\mathrm{Hz})$ & Sensor \\
\hline HSS DW & BPFI & 230.6 & AN7 \\
HSS DW & FTF & 8.6 & AN7 \\
IMS DW & BPFO & 71.8 & AN6 \\
IMS UW & BPFI & 51.7 & AN6 \\
PLC UW & BPFO & 5.9 & AN5 \\
\hline
\end{tabular}

HSS, High Speed Shaft; IMS, Intermediate Speed Shaft; PLC, Planet Carrier; BPFI, Ball Pass Frequency Inner race; BPFO, Ball Pass Frequency Outer race.

TABLE 4: Damaged bearings and their corresponding frequencies for cases B and C (1800 rpm).

\begin{tabular}{lccr}
\hline Bearing label & Fault type & Frequency $(\mathrm{Hz})$ & Sensor \\
\hline HSS DW & BPFI & 345.3 & AN7 \\
HSS DW & FTF & 12.8 & AN7 \\
IMS DW & BPFO & 105.3 & AN6 \\
IMS UW & BPFI & 73.7 & AN6 \\
PLC UW & BPFO & 8.8 & AN5 \\
\hline
\end{tabular}

HSS, High Speed Shaft; IMS, Intermediate Speed Shaft; PLC, Planet Carrier; BPFI, Ball Pass Frequency Inner race; BPFO, Ball Pass Frequency Outer race.

\section{Figure Captions:}

GTP-19-1317-Fig01 FLOWCHART OF IMPROVED ENVELOPE SPECTRUM VIA FEATURE OPTIMIZATION -GRAM (IESFOGRAM).

GTP-19-1317-Fig02 REPRESENTATION OF THE WIND TURBINE COMPONENTS AND ITS MEASURED GEARBOX [4].

GTP-19-1317-Fig03 PLANETARY GEARBOX REPRESENTATION OF BEARING LOCATION AND GEAR STAGES [4].

GTP-19-1317-Fig04 CYCLIC SPECTRAL COHERENCE MAP OF SENSOR AN7 ZOOM AROUND THE BPFI FOR: (A) DAMAGED CASE, (B) HEALTHY CASE.

GTP-19-1317-Fig05 DETECTED HSS DOWNWIND BPFI FOR SENSOR AN7: (LEFT) IESFOGRAM, AND (RIGHT) IMPROVED ENVELOPE SPECTRUM.

GTP-19-1317-Fig06 DETECTED PLC UPWIND BPFO FOR SENSOR AN5: (LEFT) IESFOGRAM, AND (RIGHT) IMPROVED ENVELOPE SPECTRUM.

GTP-19-1317-Fig07 IMPROVED ENVELOPE SPECTRUM FOR DETECTED DOWNWIND FTF FROM SENSOR AN7. 
TABLE 5: Detection accuracy for each of the 5 bearing faults for each case.

\begin{tabular}{|l|c|c|c|}
\cline { 2 - 4 } \multicolumn{1}{c|}{} & Case A & Case B & Case C \\
\cline { 2 - 4 } \multicolumn{1}{c|}{} & {$[\%]$} & {$[\%]$} & {$[\%]$} \\
\hline HSS DW BPFI & 100 & 100 & 100 \\
\hline IMS DW FTF & 100 & 100 & 100 \\
\hline IMS UW BPFI & 90 & 90 & 100 \\
\hline PLC UW BPFO & 50 & 0 & 80 \\
\hline
\end{tabular}

HSS, High Speed Shaft; IMS, Intermediate Speed Shaft; PLC, Planet Carrier; BPFI, Ball Pass Frequency Inner race; BPFO, Ball Pass Frequency Outer race.

GTP-19-1317-Fig08 IESFOGRAM FOR NON-DETECTED DOWNWIND BPFO FROM SENSOR AN5.

GTP-19-1317-Fig09 IMPROVED ENVELOPE SPECTRUM FOR NON-DETECTED DOWNWIND BPFO FROM SENSOR AN5.

GTP-19-1317-Fig10 DETECTED PLC UPWIND BPFO FROM SENSOR AN5: (LEFT) IESFOGRAM, AND (RIGHT) IMPROVED ENVELOPE SPECTRUM. 